higher and that a large number of babies are at increased risk of developing hypernatraemia.

Clearly the problem is one of education of mothers beginning in the maternity unit and continuing through the advice of health visitors and family doctors. The use of written, illustrated instructions and practical lessons in the maternity unit and clinics where mothers can be shown and then practise how to reconstitute dried milk should be more widespread. Manufacturers of dried milk could improve the mixing instructions by illustrating the procedure step by step on the packet, as is done by one major manufacturer. The information on some packets that $1 \mathrm{fl}$. oz. $(28.4 \mathrm{ml})$ equals two tablespoons could probably best be discarded and replaced by a statement that quantities must be measured accurately in a measuring jug. The volumes of tablespoons are variable and the strength of the milk will vary accordingly. As infant-feeding practices are largely influenced by tradition, folk-lore, and advertising pressures considerable educational effort may be needed to avert the trend to obesity and the dangers of hypernatraemia to which many infants seem to be exposed.

I wish to thank Dr. J. H. Briscoe-Smith, medical officer of health for the City of Westminster, for permission to interview mothers attending infant welfare clinics.

\section{References}

1 British Medical fournal, 1969, 4, 515.

2 Taitz, L. S., and Byers, H. D., Archives of Disease in Childhood, 1972, 47,257 .

3 Eid, E. E., British Medical fournal, 1970, 2, 74.

- Tracey, V. V., De, N. C., and Harper, J. R., British Medical fournal, $1971,1,16$.

5 Illingworth, R. S., The Normal Child, 4th edn. London, Churchill, 1968.

- Finberg, L., and Harrison, H. E., Pediatrics, 1955, 16, 1 .

7 Finberg, L., Kiley, J., and Luttrell, C. N., Fournal of the American Medical Association, 1963, 184, 187.

8 Gauthier, B., Freeman, R., and Beveridge, J., Australian Paediatric fournal, 1969, 5, 101 .

- Simpson, H., and O'Duffey, J. O., British Medical fournal, 1967, 3, 536.

10 Skinner, A. L., Pediatrics, 1967, 39, 625.

11 Jung, A. L., and Done, A. K., American fournal of Diseases of Children, $1969,118,859$.

12 Macauley, D., and Watson, M., Archives of Disease in Childhood, 1967, 42, 485 .

18 Dugan, S., and Holliday, M. A., Pediatrics, 1967, 39, 418.

14 Holliday, M. A., Pediatrics, 1967, 39, 626 .

${ }^{15}$ Edelmann, C. M., jun., and Barnett, H. C., fournal of Pediatrics, 1960, 56, 154.

16 Colle, E., Ayoub, E., and Raile, R., Pediatrics, 1958, 22, 5

17 Brook, C. G. D., Lloyd, J. K., and Wolf, O. H., British Medical fournal, $1972,2,25$.

18 Salans, I B. Knittle, J. L., and Hirsch, J Journal of Clinical Imoestigation, 1968, 47, 153 .

19 Taitz, L. S., British Medical fournal, 1971, 1, 315.

20 Harris, L. E., and Chan, J. C. M., American fournal of Diseases of Children, $1969,117,483$.

21 Anderson, J. A. D., and Gatherer, A., British Medical fournal, 1970, $2,20$.

2 Gatherer, A., and Wood, N., Monthly Bulletin of the Ministry of Health and The Public Health Laboratory Service, 1966, 25, 126.

\title{
Occasional Survey
}

\section{Infective Endocarditis: A Changing Disease*-II}

\author{
GRAHAM W. HAYWARD
}

British Medical fournal, 1973, 2, 764-766

The structure of the vegetations is of considerable importance in relation to treatment and complications. The vegetation itself is composed of fibrin and platelets. Leucocytes are absent and the organisms are centrally placed. In the valve beneath the vegetation there is what appears to be an attempt at healing, with fibrosis and areas of calcification. ${ }^{1}$ It seems paradoxical that bacteriostatic drugs are ineffective, and yet drugs which are bactericidal in the test-tube and effective clinically probably act as bacteriostatic drugs in the body. We are accustomed to thinking that the organisms are protected by the thick fibrin-platelet layer, but in the laboratory this is easily penetrated by the concentrataion of antibiotics available clinically, so that the organisms should be killed in a few days, whereas we know that short courses of five to seven days' treatment are always ineffective." The first essential feature of healing is inhibition of growth of bacteria to diminish the stimulus to deposition of fibrin and platelets and allow the vegetation to be covered by endo-

* Croonian lecture delivered at the Royal College of Physicians of London on 18 May 1972.

\footnotetext{
St. Bartholomew's Hospital, London EC1A 7BE, and National Heart Hospital, London W.I

GRAHAM W. HAYWARD, M.D., F.R.C.P., Senior Physician
}

thelium, and it is of interest that healing can occur even if the organisms are not killed, as shown by the demonstration of viable organisms in vegetations several months after clinical cure. Removal of turbulent flow also encourages healing. Occasionally in patients with rickettsial, fungal, or other resistant infections not responding to treatment valve replacement has been carried out in the presence of uncontrolled infection. It is not always possible to remove all infected material at the time of operation, and yet postoperatively the infection will respond. The second phase of healing is invasion at the base from the periphery with phagocytes and fibroblasts, which will transform the vegetations into fibrous tissue, often calcified, and this may well cause increased valvular incompetence.

\section{Principles in Treatment}

There are certain important general principles in treatment.

(1) In addition to recovering the organism the laboratory should determine which antibiotics singly or in combination will kill it and what concentrations are needed. This is timeconsuming, often apparently unnecessary with a sensitive organism such Streptococcus viridans, but can be life-saving. It has been our practice for several years to ensure that bactericidal levels of antibiotics are present in the blood by back-titrating the serum against the patient's own organism and adjusting the dose so that a dilution of 1 in 4 is bactericidal. 
(2) Bacteriostatic antibiotics should be avoided as they almost never effect a cure and delay the institution of more effective therapy. In only two patients in our series of nearly 300 cases have we been forced to use long-term bacteriostatic drugs to achieve cure. Both were due to a highly sensitive Str. viridans, easily killed in vitro and in vivo by penicillin, and yet relapse with positive blood cultures occurred after each of multiple courses of treatment and in one after valve replacement for heart failure. They were kept on bacteriostatic drugs for periods of nine months and six months respectively, when they were bacteriologically cured.

(3) The temptation to alter treatment if the temperature does not respond in 48 hours should be resisted. It is not uncommon for the temperature to take 10 to 14 days to settle, particularly with resistant organisms such as enterococci, and if repeated intramuscular injections are given there may be a persistently low pyrexia throughout the period of treatment. If the correct bactericidal antibiotic in the correct dose is being given there is every prospect that bacteriological cure will result.

(4) Bacteriologically negative patients should be treated as if they had a resistant organism such as the enterococcus.

The choice of antibiotic is determined by the sensitivity of the organism, and penicillin still remains the most useful drug, either alone for Str. viridans infection or combined with streptomycin for enterococcal infections. In penicillin-sensitive patients a change in antibiotic may be necessary or the sensitivity manifestations may be suppressed by prednisone, which will need to be continued for six to eight weeks after the penicillin has been stopped, as otherwise they may recur. There is still uncertainty about the duration of treatment and route and frequency of administration of drugs. The arbitrary period of six to eight weeks dates from the report on the M.R.C. clinical trials published in $1951^{3}$ and similar trials by the National Research Council in America, and most would agree that with the larger doses now given a shorter period of, say, four weeks is adequate. Although there are recurring reports of successful treatment after only two weeks of treatment relapse rates are too bigh to be acceptable in a disease as serious as infective endocarditis. For the same reason oral treatment with its unpredictable blood levels of antibiotics is unsatisfactory. For many years intermittent intramuscular injection was the treatment of choice and is still widely used. The inconvenience to the patient and the difficulty in giving large enough doses in difficult cases has led to a more widespread use of intravenous infusion, changing the catheter at weekly intervals to minimize the risk of local infection, with satisfactory results. With this method two new problems have arisen, the first concerning the advantage of continuous high-level dosage over intermittent peak levels. Theoretically there could be some advantage in using intermittent injection, as the antibiotics are more effective during periods of rapid bacterial growth.

With continuous therapy it has been suggested that the bacteria could change into cell-wall-deficient types resistant to penicillin which could revert to the original type after therapy. We have not encountered this problem in any of our cases, and with good technique to avoid infection regard intravenous therapy as the treatment of choice. We have no evidence to show whether continuous or intermittent injection is best. There is no limit to the size of dose which can be given intravenously, and the question arises whether these dosages, often greatly in excess of those necessary to produce effective bactericidal levels, are beneficial or possibly harmful. If there is evidence of impaired renal function dangerously high levels can be reached, and we have on one occasion observed convulsions from penicillin toxicity. The danger is particularly great if streptomycin is being used in conjunction with pencillin for the treatment of enterococcal infections. In these patients, who are often elderly, even $0.25 \mathrm{~g}$ of streptomycin may cause eighth-nerve damage.

\section{Death and its Cause}

The changing pattern of infective endocarditis is shown clearly by the change in mortality from the pre-antibiotic level of $100 \%$. In the first five years of the antibiotic era an early mortality of 30 to $55 \%$ was commonly reported, and similar figures are quoted now, more than 25 years after antibiotics became available. These figures are not so depressing as they appear to be at first sight, as most series include cases of acute bacterial endocarditis and surgically induced endocarditis, which is a completely different disease from the subacute or chronic endocarditis, and also bacteriologically negative cases, where the endocarditis is terminal or secondary to diffuse systemic disease. A clearer pattern emerges if we consider only bacteriologically positive cases. In my own series of nearly 300 cases the mortality before 1956 was $27 \%$, from 1956 to $196519 \%$, and from 1966 to $197214 \%$, whereas in the same period for the whole group, including surgical and bacteriologically negative cases, the figure was $31 \%$.

Another major change is in the cause of death. In 1956 uncontrolled infection was commonest, followed in order by heart failure, embolism or mycotic aneurysm, and uraemia. In 1972 heart failure was the major cause of mortality, and if the organisms can be recovered in the subacute and chronic cases failure to control the infection should rarely occur if first-class bacteriological advice is available.

If we look into the future to see how results can be improved it seems clear that they must come from prevention and treatment of heart failure. Embolism is unpredictable as a cause of mortality and occurs in the pretreatment, treatment, and early post-treatment period. Uraemia is the least common of the three major causes of mortality, and the control of the underlying chronic glomerulonephritis may rest with the immunologist. The two factors responsible for failure are the myocardial and the valvular. The increasing age of patients, many of whom have arteriosclerotic heart disease, often with atrial fibrillation, the effect of toxaemia and anaemia, and the prevalence of coronary emboli are obvious causes of myocardial failure. Valvular incompetence, with which should be included rupture of a sinus of Valsalva aneurysm, is the commonest and most important cause of heart failure. It occurs in two quite distinct ways. The first is due to fibrosis, distortion, and calcification of a valve during the processes of healing of the vegetations and causes progressive although chronic left ventricular failure, both mitral and aortic valves being equally affected. The second and more important is rupture or disruption of a valve cusp.

Anyone working in association with a cardiac surgical centre will have been struck by the great increase in cases needing urgent valve replacement in the past few years. Cusp rupture, nearly always affecting the aortic valve, occurs either during the active phase of infection or soon after the completion of treatment and is clearly distinguishable from the chronic left ventricular failure due to valvular incompetence, which develops over a period of months and is a traditional feature of the disease. It is an interesting speculation that the sudden destruction of organisms in the vegetations produced by large doses of antibiotics could produce a reaction somewhat analagous to the Herxheimer reaction, with central necrosis of the vegetations before the more chronic processes of healing and fibrosis have time to occur. The situation is not unlike that which occurred when prednisone was under trial for the treatment of rheumatic fever and was, in error, given to three patients who, in fact, had infective endocarditis. In each case valve disintegration oc- 
curred within three to seven days, with multiple emboli, free aortic regurgitation, and death. The aortic valve is far more commonly affected than the mitral, and acute left ventricular failure occurs with a clinical picture which is not always well recognized. Intense dyspnoea from pulmonary oedema is associated with a low output state and cold, sweaty extremities. Diastolic blood pressure is low and equals the left ventricular end diastolic pressure. Heart size may not be greatly increased at the onset, as the ventricle has not had time to dilate, and the early diastolic murmur of aortic regurgitation may be soft or absent. Surgical treatment of these patients has produced most gratifying results and is the major new development in the treatment of infective endocarditis.

Fears of operating during or soon after infection have proved to be groundless, and if an aortic valve has been so damaged as to cause acute or chronic left ventricular failure it should be replaced without delay, and urgent valve replacement even in the most desperate circumstances may give surprisingly good results. In those cases affecting the aortic valve careful inspection of the mitral valve is necessary, as the regurgitant jet may have caused vegetations to form on the anterior mitral valve leaflet, and this also will need surgical repair. Rupture of a sinus of Valsalva aneurysm, usually into the right atrium or ventricle, produces an unmistakable clinical picture, with a large left to right shunt and the typical loud continuous murmur maximum over the lower sternum. Urgent surgical correction is necessary, as otherwise death from heart failure may occur. There is usually less urgency with regard to the mitral valve, and surgical repair of the cusps or chordae may be possible and obviate the need for valve replacement.

\section{Prophylaxis}

True prophylaxis of infective endocarditis is possible if the underlying abnormality of the heart can be completely relieved, and this is possible in two instances only-ligation of a patent ductus and closure of a ventricular septal defect. The usual type of ventricular septal defect to be infected is the small ventricular septal defect, and surgical closure is not necessary on haemodynamic grounds and, indeed, spontaneous closure can occur. The chance of infective endocarditis developing is small and surgical correction even in the best hands carries its own mortality, so that routine prophylactic closure is not necessary. Some of these patients seem particularly prone to recurrent attacks of infective endocarditis, and in these cases closure should be carried out. Other surgical procedures to correct congenital and acauired defects always leave some cardiac abnormalities, and risk of subsequent infective endocarditis is not eliminated. Routine complete dental clearance to remove the risk of infective endocarditis is unnecessary and unsuccessful; this disease is not infrequent in the edentulous, and ill-fitting dentures causing mouth ulceration produce a new hazard.

The value of antibiotic prophylaxis is still, after 25 years of use, unproved. No figures are available on the incidence of infective endocarditis in patients at risk but it must be extremely small, considering the enormous number of potential victims who have dental extraction or other procedures without any form of antibiotic cover. Equally we do not know how effective prophylactic antibiotics are, as only a very small minority of patients develop infective endocarditis after surgical procedures which cause bacteraemia, and in two-thirds of patients the disease occurs without precipitating cause. It is probable that the variable immunological response to the infection rather than the bacteraemia itself is the more important factor. However, in our present state of knowledge it seems obligatory to use prophylactic antibiotics for those at risk. The period of bacteraemia after these surgical procedures is short and prophylaxis should be planned to cover this period only, so that with dental extractions we use 600,000 units of penicillin by injection two hours before the extraction. Longer preliminary treatment is dangerous, as an overgrowth of penicillin-resistant organisms may have occurred. For possible enterococcal infection following operations in the pelvis ampicillin is used, as the possible toxic effects of streptomycin (used with penicillin in treatment) are a greater risk than that of the disease occurring.

\section{Conclusion}

I hope I have managed to illustrate in this lecture the way in which this disease, so well described by Osler and Horder, has changed its pattern.

(1) The mortality before antibiotics of $100 \%$ has now fallen to $30 \%$, and in bacteriologically positive cases $15 \%$.

(2) The age incidence of the disease has increased and most patients now are over the age of $\mathbf{4 0}$.

(3) Because of the increasing age more patients have evidence of arteriosclerotic heart disease with aortic valve involvement, although rheumatic heart disease still provides the majority of cases.

(4) The bacteriological flora has altered, with decreasing numbers of patients infected with Str. viridans and more with other microaerophilic or anaerobic streptococci or unusual organisms.

(5) A changing view of pathogenesis emphasizes the increased importance of immunological factors in causing both the vegetations and the diffuse vascular lesions which were formerly thought to be microembolic.

(6) The symptomatology has changed. Fewer patients have overt emboli and more have diffuse and general symptoms, particularly related to the central nervous system and the musculoskeletal system.

(7) Earlier surgical treatment of valves damaged by infective endocarditis is playing an increasing part in lowering the mortality from heart failure.

I should like to express my thanks to Professor R. A. Shooter, of the department of bacteriology at St. Bartholomew's Hospital, and to my surgical colleaoues Mr. Donald Ross and Mr. Keith Ross, of the National Heart Hospital, and Mr. O. S. Tubbs and Mr. I. M. Hill, of St. Bartholomew's Hospital, whose help in the diagnosis and treatment of these patients has been so readily given.

\section{References}

1 Moore, R. A., fournal of Laboratory and Clinical Medicine, 1946, 31, 1279.

2 Beeson, P. B., in Proceedings of National Symposium on Bacterial Endocarditis, ed. P. B. Beeson and M. Ridley. London, Beecham Laboratories, 1969 .

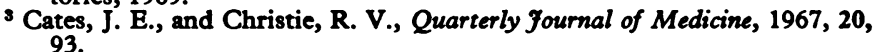

